\title{
Mitochondriopathies and bone health
}

\author{
Cosso Roberta ${ }^{1}$ and Falchetti Alberto ${ }^{1,2 *}$ \\ ${ }^{1}$ Unit of Bone and Mineral Metabolic Diseases, EndOsMet, Villa Donatello Private Hospital, Florence \\ ${ }^{2}$ Villa Alba Clinic, Villa Maria’s Group, Bologna, Italy
}

\begin{abstract}
Mitochondrial disorders represent a heterogeneous group of clinical syndromes sharing energy deficit of the mitochondrial metabolism. Although still today mitochondrial disease generally means a disorder with the oxidative phosphorylation deficiency, new knowledge of human mitochondrial genetics, biology and pathophysiology suggest the possibility of a broader spectrum of alterations and, accordingly, of presentations/clinical features. In this brief review, we will introduce the concept of why it is appropriate to write about mitochondrial disorders and bone health, passing through general concepts of mitochondrial biology and pathophysiology, because recent evidence connect the mitochondrial function to the physiology of bone cells, especially the bone-resorbing cells named osteoclasts, but not only. A deeper knowledge of mitochondrial pathophysiology in bone cells may provide one fundamental key for understanding the pathogenesis of metabolic pathways underlying skeletal diseases, both in "bone modeling" (childhood, adolescence, young adults) and in "bone remodeling" (adult, post-menopause women, elderly), and especially for the development of new therapeutical options to treat metabolic bone diseases, such as osteoporosis, and also other metabolic bone disorders, reducing the fragility fracture risk.
\end{abstract}

\section{Introduction}

Mitochondria have been described as "granules" in muscles cells by Albert von Kölliker, in 1857. Lately, other scientists also noticed these "granules" in other cell types and in 1886, the cytologist Richard Altman enabled the identification of these organelles by a dye technique, and named them "bioblasts", suggesting being the basic units of the cellular activity. Finally, at the end of nineteen century, Carl Benda coined the term mitochondria. In recent decades, mitochondria received a growing attention since in all the medical disciplines a frequent random encounter with mitochondria, with natural development of research programs, is possible.

\section{Mitochondrial genome and its differences with respect to the nuclear genome}

The mitochondrial genome, sizing $16.6 \mathrm{~kb}$ versus $3300 \mathrm{Mb}$ of the nuclear genome, consists of one circular DNA molecule, with several thousands of total numbers of DNA molecules per cell. Nuclear genes are approximately 20.000 , while mitochondrial genes are only 37 , with a gene density of $1 / 0.45 \mathrm{~kb}$. Repetitive DNA represents only a small fraction in mitochondrial DNA, with a continuous transcription of multiple genes. The 13 mitochondrial genes coding for proteins, encode all the system components of the respiratory chain, OXPHOS [1]. Intron sequences, found in most nuclear genes, are absent in mitochondrial DNA and the percentage of coding DNA is more than 90\%. Differently than nuclear DNA that presents several classes of histone and nonhistone associated proteins, the mitochondrial DNA (mtDNA) is largely free of proteins. No evidence of recombination has been reported for mitochondrial DNA and the inheritance is exclusively maternal.

\section{Nuclear and mitochondrial genes cooperation and interactions}

Mammalian mitochondria possess DNA repair pathways involving proteins, encoded by nuclear genes, which are conveyed to the mitochondria. Interestingly, many proteins involved in mitochondrial biogenesis, and in particular the proteins of the oxidative phosphorylation (OXPHOS) chain, are expressed by nuclear genes. Despite clinical and genetic data on alterations due to mtDNA mutations, few is known about human diseases/syndromes due to mutations in nuclear genes associated with the respiratory chain. It can be assumed that this will depend on the rarity of some syndromes, the genetic heterogeneity, and the incomplete knowledge of the nuclear gene kit required. Hopefully, transcriptome and proteome analysis and functional complementation studies will identify new nuclear genes related to OXPHOS, with mutations in different clinical syndromes.

\section{A. Signaling between the nucleus and mitochondria}

The existence of signaling pathways is not limited only to oxidative stress, repairing of DNA damage, and mitochondrial dysfunction, but they occur also during normal cellular metabolism. Both anterograde and retrograde signaling has been described. Anterograde signaling concerns transcription/translocation of gene products involved in mitochondrial biogenesis as also responses to stress through the antioxidant response by regulating expression of genes involved in glycolysis and calcium metabolism. Also retrograde signaling is important especially in stress, with changes levels of nicotinamide adenine dinucleotide (NAD or $\mathrm{NADH}$, depending on the state of oxidation), of reactive oxygen species (ROS), of intracellular calcium and ATP/AMP levels, and in the maintenance of the membrane potential [2]. The understanding of all these functions and interactions may be helpful to unravel potential substrates, pharmacologically treatable $[3,4]$. These future knowledges will offer an unusual number

${ }^{\star}$ Correspondence to: Falchetti Alberto, MD, Head of Metabolic Bone and Mineral Diseases, Villa Donatello Private Hospital Florence, Italy, Tel: +39331682204; E-mail alberto.falchetti2@alice.it

Key words: mitochondrial diseases; mitochondrial DNA; Bone metabolic disorders; Osteoporosis; Genetics of osteoporosis; citrate metabolism.

Received: April 04, 2018; Accepted: April 23, 2018; Published: April 27, 2018 
of important opportunities and challenges to be translated into therapeutic interventions [5].

\section{Mitochondrial functions and biology (not only the production of energy)}

Mitochondria respond to neuroendocrine, metabolic, and genetic signals through functional and morphological changes that represent signals affecting a large number of cellular functions that contribute to the complexity of the disease. As an exemplification, we can consider mitochondria as a sort of "gate" at the intersection between cell and its environment. Their biological roles can be summarized as it follows: a) remodeling of nuclear transcription; b) mitochondrial dynamics and quality control; c) inter-mitochondrial communication and inter-cellular mitochondria transfer; d) regulation of inflammation; e) immune and brain functions; and f) modulation of systemic physiological processes across organ systems. More specific roles can be summarized as: g) apoptosis and cell death for toxicity from glutamic acid; h) cell cycle regulation; i) regulation of the cell redox state; $j$ ) heme and cholesterol synthesis; $\mathrm{k}$ ) body heat production; 1$) \beta$-oxidation of fatty acids. Finally, mitochondria also have a storing function for calcium ions in the mitochondrial matrix.

\section{A. Hierarchical organization of mitochondria functions}

In recent years, a multilevel organization of molecular mitochondrial composition/structure/functions and signaling roles within the cell have been revealed. All this can be summed up in a three-dimensional hierarchy ranging from the most basic functions (first dimension) to those more complexes (second and third dimension). Specifically, aspects of the mitochondrial and cellular functions can be grouped in the first dimension, physiological functions in second dimension, and functions of systems, contributing to mortality and clinical outcomes, are present in the third dimension [6].

\section{Mitochondrial diseases}

They represent a heterogeneous group of clinical syndromes linked by an energy deficit of the mitochondrial metabolism. Although the mitochondrion is the site of various fundamental metabolic pathways, the mitochondrial diseases, in the narrow sense, are defined as syndromes associated with the deficit of OXPHOS. Since the mitochondria are present in all tissues, mitochondrial diseases can theoretically affect any organ. More often, however, they affect muscle and nerve cells, given the increased ATP demand of these tissues, especially during the development.

Since mitochondria are highly located at the neuro-muscolar level, mitochondrial diseases are often referred to as mitochondrial neuromyopathies. Mitochondrial diseases can be caused by mutations in mitochondrial DNA. Some of them affect a particular organ (for example the LHON disease, Leber's inherited optic neuropathy, which basically involves the eye), but many others concern an entire system and often present with associated neuro-myopathies. These diseases can occur at any age, with a greater incidence in that of pubertal development, due to greater energy consumption in this life's period. Generally, from a biochemical phenotype point of view, the concentration of lactic acid in the serum of patients with mitochondrial myopathies is higher since lactic acid accumulates in the muscular tissues when mitochondrial respiration is insufficient (Table 1).

\section{A. Mitochondrial diseases classification}

The most commonly used classification of mitochondrial diseases is genetic, but its main limitation lies in the fact that not all patients
Table 1. Examples of established human defects in mitochondriopathies

\begin{tabular}{|l|l|}
\hline System/sensus/organ & Clinical features/symptoms \\
\hline Central Nervous System & $\begin{array}{l}\text { migraine, psychomotor delayed/regression, dementia, } \\
\text { myoclonus, hemiparesis, ataxia, seizures, dystonia, } \\
\text { parkinsonism, hemianopia, cortical blindness, tremor }\end{array}$ \\
\hline System & peripheral neuropathy \\
\hline Muscle & $\begin{array}{l}\text { weakness, ophthalmoplegia, palpebral ptosis, exercise } \\
\text { intolerance, myoglobinuria }\end{array}$ \\
\hline Sight & pigmentary retinopathy, cataracts, optic atrophy \\
\hline Hearing & deafness/hearing loss \\
\hline Kidney & $\begin{array}{l}\text { malabsorption, intestinal pseudo obstruction, exocrine } \\
\text { pancreas failure }\end{array}$ \\
\hline $\begin{array}{l}\text { Endocrinopathies/ } \\
\text { dismetabolisms }\end{array}$ & Fanconi's syndrome/forms of renal tubular acidosis \\
\hline Heart & hypoparathyroidism short stature, diabetes mellitus \\
\hline Hemopoietic & cardiomyopathy, conduction disturbances \\
\hline
\end{tabular}

with mitochondrial disease are able to receive a precise genetic diagnosis. This is due to the variability of the clinical manifestations of mitochondrial diseases, which often makes it difficult to correlate a given picture to a specific genetic cause, both for the extreme rarity of some of the specific genetic alterations considered individually (many of which have yet to be discovered). Under normal conditions the mitochondrial DNA molecules are homoplasmic, i.e. they are all identical. However, a mtDNA disease mutation is often present in heteroplasmy, i.e. only a few and not all mitochondrial "chromosomes" (in example, certain muscle cells have affected mitochondria and others are not). Homoplasmy for a disease could be downright incompatible with life. Subjects with heteroplasmy for a disease may be affected or carriers. In fact, the disease is clinically manifested only when the number of chromosomes mutated mitochondrial reaches a certain level, called threshold of mutational load. Nevertheless, two major nosological groups can be summarized according to whether the genetic defect is localized in mitochondrial DNA (sporadic or matrilineal heredity) or in nuclear DNA (Mendelian inheritance transmission). However, we can distinguish patients with mitochondrial disease in some large "groupings" that bring together similar subjects from the clinical point of view and of interest of different organs or apparatuses, but they can present different mutations, also in different genes. Such a subdivision of patients (together with the classification by clinical phenotype) may allow considering even those suspected cases of mitochondrial disease in which, however, no genetic mutation has yet been identified. Finally, a similar classification for "apparatuses" can also be useful from an operational point of view, allowing a division of patients for the necessary "intensity of care". More recently, a growing body of research has begun to discover aspects of mitochondrial biology, beyond the production of energy, including transcriptional remodeling within the nucleus, mitochondrial dynamics and quality control, inter-mitochondrial communication, inter-cellular transfer of mitochondria, mitochondrial regulation of inflammatory processes and immune function, mitochondrial regulation of brain functions and modulation of systemic physiological processes through organ systems. Mitochondrial functions respond to a number of genetic, metabolic, neuroendocrine signals undergoing functional and morphological changes and in turn generate signals that influence a large number of cellular functions that contribute to the complexity of the disease. These issues place the mitochondria in a privileged position, like a "portal" at the intersection of the cell and its environment. Since they contain numerous potentially pharmacologically traceable components, mitochondria provide an unusual number of opportunities, and 
challenges, for translating discoveries resulting in therapeutic interventions [3-5].

\section{Why to talk about mitochondria and bone?}

It is important point out immediately that subjects affected by primary or secondary mitochondrial diseases are very fragile patients in general, in whom we should try to limit/avoid bone fragility fractures too. Recent evidence links mitochondrial function to the activity of bone cells, especially osteoclasts, but not only. In fact, bone cells have a large number of mitochondria and mitochondrial dysfunction may contribute to bone damage. Thus, mitochondria of bone cells can provide a key to understanding the pathogenesis of bone metabolic diseases, both in the modeling phase (childhood, adolescence, young adults) and in bone remodeling (adult, post-menopausal woman, elderly), and for the development of new treatment options.

\section{Are there any risk factors for a reduced bone health in mitochondrial diseases?}

The prevalence of risk factors for poor bone health in primary mitochondrial diseases is high. Despite the considerable variability in clinical presentation, individuals with mitochondrial disease may be at risk for reduced bone health, especially if they are chronically ill, malnourished and/or undergone prolonged immobilization. As part of a supportive care, physicians should begin to study modifiable risk factors to optimize bone health, with a low threshold for the assessment of clinical symptoms that may suggest an occult fragility fracture. Mitochondrial disease can also increase the risk for endocrine conditions that are known to negatively affect bone quantity and quality, including diabetes mellitus, hypoparathyroidism, growth hormone deficiency and hypogonadism, as well as other diseases that increase the risk of osteopenia and osteoporosis, such as renal tubular acidosis and acute or chronic liver disease [7,8]. Currently, our understanding of bone health in mitochondrial diseases is limited and stems primarily from case studies of children with mitochondrial diseases and impaired bone health. For example, a five-year-old boy with Kearns Sayre syndrome (KSS) was described to develop rickets with bone deformities and fractures, with bone health deteriorating to such an extent that he lost the ability to walk [9]. Despite the significant potential burden of osteopenia and osteoporosis in this population, and the clinical availability of systemic anti-osteoporotic therapies, bone health studies in mitochondrial disease are deficient $[10,11]$.

\section{Mitochondria and aging}

Therefore, it clearly appears that alterations of the mitochondria, in the broadest sense of the term, can contribute to the pathogenesis of other pathological conditions, in particular the degenerative ones that most afflict the aging population. A wide spectrum of alterations in mitochondria, and in mtDNA, has been observed with aging in animals and humans. These include: 1) decrease in mitochondrial respiratory function; 2) increase in mitochondrial production of ROS and extension of oxidative DNA damage to proteins and lipids; 3 ) the accumulation of point mutations and/or large mtDNA deletions; and 4) increased rate of apoptosis. Recent studies have provided abundant evidence to validate the importance of mitochondrial ROS production in aging. On the other hand, somatic mutations of mtDNA can cause premature aging without increasing ROS production. Taking these results together, they suggested that mitochondrial dysfunction, increased oxidative stress, consequent accumulation of mtDNA mutations, altered expression of some gene groups and apoptosis are important "collaborators" in human aging.

\section{A. Mitochondria, skeletal aging, osteoporosis, and oxidative stress}

It is well known that there is a reduction in the number of osteocytes due to the increase in age-dependent oxidative stress. The production of $\mathrm{H}_{2} \mathrm{O}_{2}$, and numerous other ROS, generated during aerobic metabolism within the mitochondria, increases with advancing age. This increase is amplified by the phosphorylation of p66, which oxidizes the reduced "mitochondrial" cytochrome-c, released by the electron transfer chain and opens the permeability transition pore, leading to apoptosis. Cells attempt to counteract the adverse effects of oxidative stress with different mechanisms, including an increase in different types of autophagy, stimulated by the activation of transcription factors such as FoxOS and NF-kB. The failure of autophagy with age further contributes to the disappearance of cells. Furthermore, as we age, cells with long postmitotic life are unable to maintain the nuclear pore diffusion barrier, resulting in "leakiness" caused by the oxidation of nucleoporins, such as Nup-93. Free radicals are also involved in "osteopathic" apoptosis and osteoclastogenesis and, therefore, in bone resorption, as shown in several in vitro and animal studies [12]. Finally, we well know how the oxidative stress antagonizes the Wnt pathway, necessary for proper osteoblastogenesis, in osteoblast precursors (mice). Oxidative stress is therefore an important pathogenetic factor of bone loss related to age and insulin-resistance, quite common in the older and obese population, with a reduction in the number of osteoblasts and, consequently, in bone formation. Situations characterized by an excessive accumulation/formation of glucose (hyperglycemia) products containing ROS and AGEs have negative effects on the maturation of collagen molecules, altering their stability and chemical-physical properties, such as elasticity and deformability. The pathway of collagen cross-linking depends on the activities of lysine-hydroxylase and lysyl-oxidase regulating the reaction (enzymatic crosslinking). The first step, at all ages, is the non-enzymatic glycation, oxidation, or glycoxidation between lysine or hydroxylisine residues and the sugar. The non-enzymatic cross-link formation with AGEs deteriorates bone biological and mechanical functions. Mesenchymal stem cells (MSC) of bone marrow, osteoblasts and osteoclasts, express AGEs receptors, called RAGEs, which can bind AGEs with high affinity, altering the normal cell homeostasis. AGE/RAGE binding reduces the osteogenic potential of MSC, inhibits the ability to osteoblastic bone formation and induces a long-term decrease in recruitment and bone resorption activity of osteoclasts. Overall, these cellular effects of AGEs depress the bone turnover and, therefore, induce an even greater accumulation of the AGEs themselves. Beyond the clinical risk factors, there may also be a direct negative effect of mitochondrial disease on the level of maturation and skeletal turnover. Osteoclasts are rich in mitochondria and the availability of energy seems to affect their resorbing abilities. The activity of cytochrome-c oxidase, complex IV of the mitochondrial respiratory chain, has been indicated to regulate the osteoclast activity necessary for adaptive bone remodeling [13].

\section{Mitochondrial defects in animal and human models}

Mice expressing a defective mitochondrial DNA polymerase (PolgA) are models of the corresponding disease in humans. Homozygous knock-in mice with a deficient version of PolgA (a catalytic subunit of the mitochondrial DNA polymerase) develop kyphosis and osteoporosis. In particular, in a murine model of mitochondrial diseases PolgA related, osteoblasts with respiratory chain dysfunction were found and the animals had poor bone health [14,15]. Another approach to understanding the role of mitochondria in bone health is the search for bio-energetic defects in apparently healthy individuals 
with pathological fractures. One study examined the mitochondrial genome from 15 blood samples from male subjects with vertebral fractures and found a new $3.7 \mathrm{~kb}$ mitochondrial DNA deletion, which included genes encoding the subunits of the mitochondrial respiratory chain complex I, in two of these patients [16]. It could also be suggested that osteoporosis due to aging may be partly attributable to the accumulation of heteroplasmic mitochondrial DNA mutations. In a recent study, the authors were unable to isolate the direct effects of mitochondrial dysfunction on bone from the indirect effects of mitochondrial dysfunction on bone depending on related comorbidity and drug [17]. Moreover, fractures were present sustained in $14 \%$ of the included subjects. In 55\% of cases, a first fracture occurred before the age of 9 years. Age distribution and profile exposure of any cohort will substantially influence the predicted fracture rate, as shown by the incidence of senile fracture in the reference populations [18]. However, according to the growing body of literature on the role of mitochondria in skeletal health, we might expect that mitochondrial dysfunction exerts an independent, negative effect on bone. Future studies will have to address this important problem. Although there are currently no other large studies reporting fractures in individuals with mitochondrial disease, fracture rates can be examined for comparison in other diseases. For example, even patients with Friedreich's ataxia (FRDA) develop neurological disease, diabetes mellitus, and limited mobility. In a study of 28 subjects with FRDA, it is described that $21 \%$ had a history of bone fractures and the same percentage had a Dual X Rays Absorptiometry (DXA) Z score $\leq-2.0$ SD of bone mineral density (BMD), that is frankly reduced than expected. [19]. A retrospective study with 221 patients with "spina bifida" (age 2-58 years) has documented 42 fractures, obtaining a similar rate as observed in a recent study ( $19 \%$ vs. $20 \%$, respectively) [20]. Similarly to individuals with mitochondrial myopathies, boys with Duchenne's muscular dystrophy (DMD) have reduced muscle strength that can predispose to falls, as well as fractures and poor bone health. A "cross-sectional" study in DMD (mean age 12 years) shows a fracture rate of $21 \%$ in life [21]. These studies suggest relatively comparable rates of reduced bone health and fractures in other conditions. The rate of excess epilepsy in mitochondrial disease deserves a separate consideration. Patients with epilepsy have multiple risk factors for osteoporosis and fractures, including falls induced by epilepsy, anti-epileptic drugs, immobility, low body mass index (BMI) and increased prevalence of endocrine comorbidities, including growth hormone deficiency and hypothyroidism [22]. A recent study evaluated risk factors for reduced bone health in 260 patients with epilepsy (not necessarily related to mitochondrial disease) and found that $11 \%$ had osteoporosis and $38 \%$ had at least one fracture in life [23]. Fifty-two percent of those patients who underwent bone mass assessment, the DXA analyses show a reduced BMD. A significant proportion of patients with primary mitochondrial disease have an important spinal curvature, which can also affect BMD and its clinical evaluation. Many studies have suggested that as spinal curvature increases the BMD decreases $[24,25]$. The presence of spinal curvature should be considered when considering risk factors for reduced bone health.

\section{Mitochondria and citrate}

It is well known that citrate is mainly produced in the mitochondria via glucose-derived pyruvate. In the mitochondria, citrate is oxidized via the Krebs cycle and OXPHOS. Citrate represents a key substrate for energy generation and acts as an allosteric modulator of several enzymes as well. The high citrate concentration into cytoplasm modulates the lipid synthesis and blunts glycolysis by inhibiting phosphofructokinase-1 (PFK1), a rate-limiting enzyme of glycolysis
$[26,27]$. The ion transporter SLC25A1, or CIC, is embedded in the inner mitochondrial membrane, and CIC defects have been indirectly linked to various human diseases, such as including diabetes, neurodegeneration, aging, potentially negative for bone quality/strength, as also cancer [28]. CIC determines the efflux across the mitochondria of tricarboxylic citrate in exchange for dicarboxylic cytosolic malate, important step for a good modulation of cell metabolism and viability. Thus, malate enters into the mitochondria by exchanging with citrate and it enters into the Krebs cycle in place of citrate, also stimulating OXPHOS [27]. Therefore, the malate-citrate import-export pathway highly impact on mitochondrial viability. It has been reported that citrate supplementation may negatively affect the glycolytic pathway, and changes in the energy systems affect differentiation and function of osteoclast $[29,30]$. Moreover, citrate is able to bind calcium and consequently it may reduce the $\mathrm{Ca}^{2+}$ availability, interrupting also the Nuclear Factor of Activated T-cells cytoplasmic 1 (NFATc1) signaling (NFATc1 is a component of the nuclear factor of activated T cells DNAbinding transcription complex) activated by the proton excess, and it has been suggested that intracellular $\mathrm{Ca}^{2+}$ chelation hampers the osteoclast survival induced by Receptor Activator of Nuclear factor Kappa-B Ligand (RANKL), known to control bone regeneration and remodeling [31-33]. As also above indirectly suggested, it is known that an excessive acid load is physiologically back to balance by a metabolic adaptation involving primarily kidney, lung, and bone [34]. At skeletal level, an acidic microenvironment may affect bone cells facilitating a higher resorption rate of the mineralized matrix to recruit hydroxyl groups able to neutralize the proton excess when the overall buffering capacity is limited, but when acidic overloading persists, osteopenia due to the net loss of calcium and deterioration of microarchitecture may appear [35]. Thus, oral administration of alkali salts, such as $\mathrm{K}^{+}$citrate has been proposed to counterbalance the low-grade acidosis, preventing bone loss. Unfortunately, it has not been clearly defined if the expected beneficial effects could due to the alkalizing function of $\mathrm{K}^{+}$citrate or to an its direct influence on bone cell activity, even if preliminary clinical data are encouraging [36-42]. Treatment with $\mathrm{K}^{+}$citrate for 24 months of a group of healthy elderly persons without osteoporosis, showed a significant increase in both areal and volumetric BMD in the several sites investigated, also improving bone microarchitecture. Based on the effect on fracture prediction, a possible protective effect on future fractures by $\mathrm{K}^{+}$citrate might be hoped [41].

Very recently, in a study on primary culture of bone cells, it has been showed that $\mathrm{K}^{+}$citrate inhibits osteoclastogenesis and enhance the anti-osteoclastogenic activity of alendronate, an amino-bisphosphonate commonly used in osteoporotic subjects since it reduces vertebral, nonvertebral and femoral risk fracture. Interestingly, such effect did not seem depend only on alkalizing properties of $\mathrm{K}^{+}$citrate. If such findings will be replicate and confirm in further studies, including on humans, they could provide a biological basis for a clinical use of $\mathrm{K}^{+}$ citrate in order to prevent the bone loss occurring in low-grade acidosis state, and to deeply investigate an eventual anti-osteoclastogenic role of $\mathrm{K}^{+}$citrate [43]. Wider randomized multicentric clinical studies are needed to definitively assess the dosage schedule, timing and duration of supplementation, and to confirm effective benefits of $\mathrm{K}^{+}$citrate in preventing the osteopenia progression.

\section{Mitochondrial risk factors for a poor bone health: secondary mitochondriopathies}

While the term of mitochondrial disorder usually refers to primary disorders of mitochondrial metabolism affecting OXPHOS, it has to be taken also into account that mitochondrial function disorders are 
not observable only within specific genetic disorders, but they can be also seen as part of the aging process and/or multifactorial metabolic disorders, such as chronic inflammatory diseases, diabetes, metabolic syndrome, cardiovascular diseases, and human cancers. These are not strictly mitochondrial diseases.

\section{A. Damage induced by deficiencies and/or use of drugs with negative impact on bone health}

Since vitamin D deficiency is a modifiable risk factor for poor bone health, vitamin $\mathrm{D}$ reposition strategies may require more dedicated attention, with individualized titration in this population [44]. Furthermore, there may be advantages, beyond the bone health, for adequate levels of vitamin $\mathrm{D}$, including improved ability of oxidative muscle phosphorylation [45]. Diabetes mellitus is another major risk factor for osteopathy and one of the most common primary endocrine manifestations of mitochondrial disease [46,47]. Multiple mechanisms have been suggested for the association between DM and osteopathy. For example, the accumulation of AGEs can cause osteoblast apoptosis and decrease bone formation [48]. In affected patients, special attention to diabetes management, including surveillance for potential bone effects, must be ensured. Many drugs increase the risk of osteoporosis [49]. Systemic glucocorticoids and anticonvulsants have often been used in individuals with mitochondriopathies and each of these drugs has been shown to have deleterious effects on bone turnover [50]. Abnormal bone turnover can adversely affect bone quality so that it is not reflected in the BMD as assessed by DXA but may increase the risk of fracture.

\section{Author's suggestions and future perspectives}

Mitochondrial diseases represent a heterogeneous group of disorders ranging from neuro- myopathies, to endocrine disorders, and dismetabolisms. Subjects affected with primary mitochondriopathies, generally pediatric or young adult subjects, may present with severe major endocrine-metabolic alterations, difficulties in deambulation or with severe immobility due to neuro-myopathies, severe renal failure and/or alteration of the acid-base state, that is all of them are very fragile subjects. Consequently, these same pathological changes may also have a negative impact on skeletal health and bone tissue also represent a target of this general fragility. It's easily understandable how the occurrence of fragility fractures, even at skeletal sites less frequently involved, represents a further progression in the negative sense of an already compromised quality of life, contributing to reduce the life span. Even if primitive mitochondriopathies are rare disorders, we know that rare diseases are essential to understand how many common diseases develop and how they could be prevented or treated. While, regarding the primary mitochondrial diseases for their correct identification/diagnosis we have sophisticated techniques of molecular genetics, DNA sequencing and immunohistochemical investigations on tissue biopsy, unfortunately in secondary mitochondrial diseases, potentially representing an important percentage of fragile bones occurring in postmenopausal women and/or elderly adult, the above methodologies cannot be routinely applied with a significant sensitivity and specificity. In particular, we can hardly find significant increases in plasma concentrations of lactic acid, significantly deriving from skeletal muscles. Certainly, however, we strongly recommend all the various specialists who may be faced with patients with mitochondrial disorders, to carry out the current diagnostic protocols for a clinical evaluation of brittle bones, such as peripheral DXA scan, bone and mineral turnover, skeletal $\mathrm{x}$-rays and so on, or alternatively to refer to the help of bone specialist [51].
Currently, bone anti-resorptive and/or anabolic therapies can be considered for patients with severe osteopenia or osteoporosis, but we don't have a clear body of evidence, in randomized clinical trials, for their use in patients with mitochondrial disorders, neither primitive nor secondary. Specifically, amino-bisphosphonates are a mainstay of osteoporosis therapy, but there is limited evidence of underlying mitochondrial disease, particularly in children. Vesicle trafficking pathways have a role in regulating mitochondrial quality control and loss of trafficking mechanism impairs the ability of mitochondria to selectively degrade oxidized and damaged proteins leading, over time, to the mitochondrial dysfunction as reported in subjects affected by Parkinson's disease [52]. Thus, vesicle trafficking mechanism can be regarded as pharmacological targets. In particular, vesicular trafficking is critical for the function of bone cells, as exemplified by bone diseases such as osteopetrosis, which frequently results from defects in this process [53]. Modulators of endoplasmic reticulum stress themselves have shown potential as treatment options in osteoporosis. [54] Aminio-bisphosphonates disrupt osteoclast-mediated bone resorption by inhibiting the function of Rab GTPases, that physiologically regulate many steps of membrane trafficking, including vesicle formation, vesicle movement along actin and tubulin networks, and membrane fusion, thereby impairing vesicular trafficking, as well as impairing other small GTPase-dependent processes [55]. Consequently, the safety, tolerability and efficacy of anti-osteoporotic therapies in subtypes, age-dependent, of mitochondrial disease are important areas for future studies. Portion of patients with early-onset osteoporosis could be regarded as highly suspicious to have rare or private pathogenic variants in one or more genes modulating mitochondrial biology and potentially involved in the regulation of bone mass. Further studies will be necessary to evaluate a possible interplay of several potentially pathogenic variants, especially if the genes belong to overlapping mitochondrial functional pathways. This could provide a basis for future for individualized therapeutic strategies for disorders with low bone mass. So, if we can understand how mitochondrial alterations can contribute to bone metabolism and to determine bone fragility, we will have new opportunities to develop specific therapeutic interventions which can be applied also to bone fragility in general and, in particular, the one linked to ageing.

Unfortunately, it is not justifiable to resort to expensive and timeconsuming molecular genetic strategies, as a population screening, and we currently have many limitations on a possible biochemical-clinical characterization specifically designed to identify mild-moderate mitochondrial abnormalities, in patients with "classical" osteoporosis and the current protocols are hardly likely to be useful for this purpose (www. mitopedia.org).

Future perspectives in mitochondrial genetic analysis will be offered by next generation sequencing (NGS) technology. The NGS approach permits simultaneous sequencing of extra-/intra-genic and mitochondrial regions. Moreover, NGS extend the sequencing of nucleotides from a single gene up to the multigene level by specifically setting up targeted panels, up to the whole genome, with huge amounts of data on a gigabyte scale in a single step. NGS reaches adequate resolution levels for any single genetic analysis, and a sequencing run can be specifically tailored to obtain genetic data and/or to screen one or more predetermined genomic regions or a specifically desired gene set [56]. Recently, analytical tools have been developed to evaluate also mtDNA in whole-exome data and recent findings provided further support for the role of mitochondria in complex human disorders, such as autism and we hope it can become even for disorders at the base of bone fragility [57]. 


\section{Authorship and contributorship}

Roberts Cosso, M.D., specialist in Orthopedics and Traumatology, gave her precious assistance in writing the manuscript and in its English revision. Moreover, she strongly contributed in focusing some important tips in highlighting potential aspect important in clinical practice; Alberto Falchetti, M.D., wrote the manuscript and made all the substantive intellectual contributions to its preparation, both as an endocrinologist expert in bone metabolism and as a medical geneticist, offering his expert international biomedical authorship, also due to his important institutional role in the Italian Society of Osteoporosis and Mineral Metabolism and Skeletal Diseases (S.I.O.M.M.M.S).

Non-financial competing interests to declare.

\section{References}

1. Alston CL, Rocha MC, Lax NZ, Turnbull DM, Taylor RW (2017) The genetics and pathology of mitochondrial disease. J Pathol 241: 236-250. [Crossref]

2. Saki M, Prakash A (2017) DNA damage related crosstalk between the nucleus and mitochondria. Free Radic Biol Med 107: 216-227. [Crossref]

3. Andreux PA, Houtkooper RH, Auwerx J (2013) Pharmacological approaches to restore mitochondrial function. Nat Rev Drug Discov 12: 465-483. [Crossref]

4. Wang W, Karamanlidis G, Tian R (2016) Novel targets for mitochondrial medicine. Sci Transl Med 8: 326rv3. [Crossref]

5. Hersh SP (2014) Fast-tracking the development of effective therapeutics in mitochondrial medicine. Clin Pharmacol Ther 96: 641-643. [Crossref]

6. Picard M, Wallace DC, Burelle Y (2016) The rise of mitochondria in medicine. Mitochondrion 30: 105-116. [Crossref]

7. Harvey JN, Barnett D (1992) Endocrine dysfunction in Kearns-Sayre syndrome. Clin Endocrinol (Oxf) 37: 97-103. [Crossref]

8. Mirza F, Canalis E (2015) Management of endocrine disease: Secondary osteoporosis: pathophysiology and management. Eur J Endocrinol 173: R131-151. [Crossref]

9. Tzoufi M, Makis A, Chaliasos N, Nakou I, Siomou E, et al. (2013) A rare case report of simultaneous presentation of myopathy, Addison's disease, primary hypoparathyroidism, and Fanconi syndrome in a child diagnosed with Kearns-Sayre syndrome. Eur J Pediatr 172: 557-561. [Crossref]

10. Cholley F, Edery P, Ricquier D, Peudenier S, Slama A, et al. (2001) Mitochondrial respiratory chain deficiency revealed by hypothermia. Neuropediatrics 32: 104-106. [Crossref]

11. De Block CE, De Leeuw IH, Maassen JA, Ballaux D, Martin JJ (2004) A novel 7301bp deletion in mitochondrial DNA in a patient with Kearns-Sayre syndrome, diabetes mellitus, and primary amenorrhoea. Exp Clin Endocrinol Diabetes 112: 80-83. [Crossref]

12. Almeida M, Han L, Ambrogini E, Bartell SM, Manolagas SC (2010) Oxidative stress stimulates apoptosis and activates NF-kappaB in osteoblastic cells via a PKCbeta/ p66shc signaling cascade: counter regulation by estrogens or androgens. Mol Endocrinol 24: 2030-2037. [Crossref]

13. Miyazaki T, Neff L, Tanaka S, Horne WC, Baron R (2003) Regulation of cytochrome c oxidase activity by c-Src in osteoclasts. J Cell Biol 160: 709-718. [Crossref]

14. Trifunovic A, Wredenberg A, Falkenberg M, Spelbrink JN, Rovio AT, et al. (2004) Premature ageing in mice expressing defective mitochondrial DNA polymerase. Nature 429: 417-423. [Crossref]

15. Dobson PF, Rocha MC, Grady JP, Chrysostomou A, Hipps D (2016) Unique quadruple immunofluorescence assay demonstrates mitochondrial respiratory chain dysfunction in osteoblasts of aged and PolgA(-/-) mice. Sci Rep 6: 31907. [Crossref]

16. Varanasi SS, Francis RM, Berger CE, Papiha SS, Datta HK (1999) Mitochondrial DNA deletion associated oxidative stress and severe male osteoporosis. Osteoporos Int 10: 143-149. [Crossref]

17. Gandhi SS, Muraresku C, McCormick EM, Falk MJ, McCormack SE (2017) Risk factors for poor bone health in primary mitochondrial disease. J Inherit Metab Dis 40: 673-683. [Crossref]

18. Weber DR, Haynes K, Leonard MB, Willi SM, Denburg MR (2015) Type 1 diabetes is associated with an increased risk of fracture across the life span: a population-based cohort study using The Health Improvement Network (THIN). Diabetes Care 38: 1913-20. [Crossref]
19. Eigentler A, Nachbauer W, Donnemiller E, Poewe W, Gasser RW, et al. (2014) Low bone mineral density in Friedreich ataxia. Cerebellum 13: 549-557. [Crossref]

20. Dosa NP, Eckrich M, Katz DA, Turk M, Liptak GS (2007) Incidence, prevalence, and characteristics of fractures in children, adolescents, and adults with spina bifida. $J$ Spinal Cord Med 30 Suppl 1: S5-9. [Crossref]

21. McDonald DG, Kinali M, Gallagher AC, Mercuri E, Muntoni F, et al. (2002) Fracture prevalence in Duchenne muscular dystrophy. Dev Med Child Neurol 44: 695-698. [Crossref]

22. Wei SH, Lee WT2 (2015) Comorbidity of childhood epilepsy. J Formos Med Assoc 114: 1031-1038. [Crossref]

23. Fedorenko M, Wagner ML, Wu BY (2015) Survey of risk factors for osteoporosis and osteoprotective behaviors among patients with epilepsy. Epilepsy Behav 45: 217-22. [Crossref]

24. Pavlovic A, Nichols DL, Sanborn CF, Dimarco NM (2013) Relationship of thoracic kyphosis and lumbar lordosis to bone mineral density in women. Osteoporos Int 24 2269-2273. [Crossref]

25. Sadat-Ali M, Al-Othman A, Bubshait D, Al-Dakheel D (2008) Does scoliosis causes low bone mass? A comparative study between siblings. Eur Spine J 17: 944-947. [Crossref]

26. Newsholme EA, Sugden PH, Williams T (1977) Effect of citrate on the activities of 6-phosphofructokinase from nervous and muscle tissues from different animals and its relationships to the regulation of glycolysis. Biochem J 166: 123-129. [Crossref]

27. Owen OE, Kalhan SC, Hanson RW (2002) The key role of anaplerosis and cataplerosis for citric acid cycle function. J Biol Chem 277: 30409-30412. [Crossref]

28. Catalina-Rodriguez O, Kolukula VK, Tomita Y, Preet A, Palmieri F, et al. (2012) The mitochondrial citrate transporter, CIC, is essential for mitochondrial homeostasis. Oncotarget 3: 1220-1235. [Crossref]

29. Kim JM, Jeong D, Kang HK, Jung SY, Kang SS, et al. (2007) Osteoclast precursors display dynamic metabolic shifts toward accelerated glucose metabolism at an early stage of RANKL-stimulated osteoclast differentiation. Cell Physiol Biochem 20: 935946. [Crossref]

30. Lemma S, Sboarina M, Porporato PE, Zini N, Sonveaux P, et al. (2016) Energy metabolism in osteoclast formation and activity. Int J Biochem Cell Biol 79: 168-180. [Crossref]

31. Kato K, Morita I (2013) Promotion of osteoclast differentiation and activation in spite of impeded osteoblast lineage differentiation under acidosis: effects of acidosis on bone metabolism. Biosci Trends 7: 33-41. [Crossref]

32. Yuan FL, Xu MH, Li X, Xinlong H, Fang W, et al. (2016) The Roles of Acidosis in Osteoclast Biology. Front Physiol 7: 222. [Crossref]

33. Komarova SV, Pilkington MF, Weidema AF, Dixon SJ, Sims SM (2003) RANK ligand-induced elevation of cytosolic $\mathrm{Ca}^{2+}$ accelerates nuclear translocation of nuclear factor kappa B in osteoclasts. J Biol Chem 278: 8286-8293. [Crossref]

34. Pizzorno J (2015) Acidosis: An Old Idea Validated by New Research. Integr Med (Encinitas) 14: 8-12. [Crossref]

35. Arnett TR (2010) Acidosis, hypoxia and bone. Arch Biochem Biophys 503: 103-109. [Crossref]

36. Lambert H, Frassetto L, Moore JB, Torgerson D, Gannon R, et al. (2015) The effect of supplementation with alkaline potassium salts on bone metabolism: a meta-analysis. Osteoporos Int 26: 1311-1318. [Crossref]

37. Vescini F, Buffa A, La Manna G, Ciavatti A, Rizzoli E, et al. (2005) Long-term potassium citrate therapy and bone mineral density in idiopathic calcium stone formers. $J$ Endocrinol Invest 28: 218-222. [Crossref]

38. Sellmeyer DE, Schloetter M, Sebastian A (2002) Potassium citrate prevents increased urine calcium excretion and bone resorption induced by a high sodium chloride diet. $J$ Clin Endocrinol Metab 87: 2008-2012. [Crossref]

39. Sakhaee K, Maalouf NM, Abrams SA, Pak CY (2005) Effects of potassium alkali and calcium supplementation on bone turnover in postmenopausal women. $J$ Clin Endocrinol Metab 90: 3528-3533. [Crossref]

40. Jehle S, Zanetti A, Muser J, Hulter HN, Krapf R (2006) Partial neutralization of the acidogenic Western diet with potassium citrate increases bone mass in postmenopausal women with osteopenia. J Am Soc Nephrol 17: 3213-3222. [Crossref]

41. Jehle S, Hulter HN, Krapf R (2013) Effect of potassium citrate on bone density, microarchitecture, and fracture risk in healthy older adults without osteoporosis: randomized controlled trial. J Clin Endocrinol Metab 98: 207-217. [Crossref] 
42. Moseley KF, Weaver CM, Appel L, Sebastian A, Sellmeyer DE (2013) Potassium citrate supplementation results in sustained improvement in calcium balance in older men and women. J Bone Miner Res 28: 497-504. [Crossref]

43. Granchi D, Torreggiani E, Massa A, Caudarella R, Di Pompo G, et al. (2017) Potassium citrate prevents increased osteoclastogenesis resulting from acidic conditions: Implication for the treatment of postmenopausal bone loss. PLoS One 12: 0181230.

44. Misra M, Pacaud D, Petryk A, Collett-Solberg PF, Kappy M, et al. (2008) Vitamin $\mathrm{D}$ deficiency in children and its management: review of current knowledge and recommendations. Pediatrics 122: 398-417. [Crossref]

45. Sinha A, Hollingsworth KG, Ball S, Cheetham T (2013) Improving the vitamin D status of vitamin $\mathrm{D}$ deficient adults is associated with improved mitochondrial oxidative function in skeletal muscle. J Clin Endocrinol Metab 98: E509-513. [Crossref]

46. Schaefer AM, Walker M, Turnbull DM, Taylor RW (2013) Endocrine disorders in mitochondrial disease. Mol Cell Endocrinol 379: 2-11. [Crossref]

47. Whittaker RG, Schaefer AM, McFarland R, Taylor RW, Walker M, et al. (2007) Prevalence and progression of diabetes in mitochondrial disease. Diabetologia 50: 2085-2089. [Crossref]

48. Khan TS, Fraser LA (2015) Type 1 diabetes and osteoporosis: from molecular pathways to bone phenotype. J Osteoporos 2015: 174186. [Crossref]

49. Perin P, Potočnik U (2014) Polymorphisms in recent GWA identified asthma genes CA10, SGK493, and CTNNA3 are associated with disease severity and treatment response in childhood asthma. Immunogenetics 66: 143-151. [Crossref]
50. Tóth M, Grossman A (2013) Glucocorticoid-induced osteoporosis: lessons from Cushing's syndrome. Clin Endocrinol (Oxf) 79: 1-11. [Crossref]

51. Lewiecki EM (2000-2015) Osteoporosis: Clinical Evaluation. n: De Groot LJ, Chrousos G, Dungan K, Feingold KR, Grossman A, Hershman JM, Koch C, Korbonits M, McLachlan R, New M, Purnell J, Rebar R, Singer F, Vinik A, editors. Endotext [Internet]. South Dartmouth (MA): MDText.com, Inc. [Crossref]

52. McLelland GL, Soubannier V, Chen CX, McBride HM, Fon EA (2014) Parkin and PINK1 function in a vesicular trafficking pathway regulating mitochondrial quality control. EMBO J 33: 282-295. [Crossref]

53. Stenbeck G, Coxon FP (2014) Role of vesicular trafficking in skeletal dynamics. Curr Opin Pharmacol 16: 7-14. [Crossref]

54. Hino S, Kondo S, Yoshinaga K, Saito A, Murakami T, et al. (2010) Regulation of ER molecular chaperone prevents bone loss in a murine model for osteoporosis. $J$ Bone Miner Metab 28: 131-138. [Crossref]

55. Itzstein C, Coxon FP, Rogers MJ (2011) The regulation of osteoclast function and bone resorption by small GTPases. Small GTPases 2: 117-130. [Crossref]

56. Forlenza GP, Calhoun A, Beckman KB, Halvorsen T, Hamdoun E, et al. (2015) Next generation sequencing in endocrine practice. Mol Genet Metab 115: 61-71. [Crossref]

57. Patowary A, Nesbitt R, Archer M, Bernier R, Brkanac Z (2017) Next Generation Sequencing Mitochondrial DNA Analysis in Autism Spectrum Disorder. Autism Res 10: 1338-1343. [Crossref]

Copyright: $(02018$ Roberta C. This is an open-access article distributed under the terms of the Creative Commons Attribution License, which permits unrestricted use, distribution, and reproduction in any medium, provided the original author and source are credited. 